\title{
Políticas ambientales en México. El caso de los contaminantes orgánicos persistentes
}

\author{
Mariana Villada Canela*
}

Resumen: ${ }^{1}$ en este artículo se examina el efecto del uso de la información en la participación y en la toma de decisiones para la elaboración, en México, del Plan Nacional de Implementación del Convenio de Estocolmo sobre Contaminantes Orgánicos Persistentes. La revisión documental, observación participante y entrevistas revelan que: a) la información es un recurso para ostentar poder técnico pero, sobre todo, político, cuando se moviliza para situar intereses específicos y b) que ese poder se refleja en la elaboración de diagnósticos y subplanes en la política ambiental. Por consiguiente, utilizar de este modo la información puede representar un medio de exclusión, cuando predomina una participación técnicamente competente o limitada a aquellos con poder económico, así como moldear la participación en la hechura del mencionado plan, y vincularlo al cumplimiento de compromisos internacionales, juegos de poder y a una exclusión no intencional, para prevalecer en el debate y en la toma de decisiones.

* Investigadora del Instituto de Investigaciones Oceanológicas, Universidad Autónoma de Baja California (UABC). Carretera transpeninsular Ensenada-Tijuana \# 3917, fraccionamiento Playitas, C.P. 22860, Ensenada, Baja California, México. Teléfono: (646) 1744601 . Correo electrónico: mvilladac@uabc.edu.mx

1 Agradezco al Consejo Nacional de Ciencia y Tecnología por la beca recibida. Este estudio se llevó a cabo en El Colegio de México, con Judith Domínguez y Vicente Ugalde como asesores. También agradezco al Instituto de Investigaciones Oceanológicas de la UABC las facilidades otorgadas y a los revisores de este artículo. 
Palabras clave: política ambiental; toma de decisiones; aspectos científico-políticos; contaminantes; información; participación.

Abstract: this article examines the effect of the use of information on participation and decision-making during the development of Mexico's National Implementation Plan (NIP) for the Stockholm Convention on Persistent Organic Pollutants. A literature review, participant observation and interviews revealed that: 1) information on persistent organic pollutants is a resource used to exert technical as well as political power when it is mobilized in favor of specific interests; and 2) this power is reflected in the development of environmental policy documents and sub-plans. As a consequence, using information in this way could be a means of exclusion, as participation requires technical competence and is limited to those with economic power. Thus, information shapes participation in the development of the NIP and is linked to the fulfillment of international agreements, but it is also linked to power games and an unintentional exclusion used by certain actors to prevail in debates and decision-making.

Key words: environmental policy; decision making; scientific and political aspects; pollutants; information; participation.

\section{Introducción}

Los avances científicos y tecnológicos también tienen efectos adversos en el ambiente y en las personas, que en ocasiones son imprevistos y que, paradójicamente, sólo son abordados por los científicos (Jasanoff 1990). En consecuencia, la política ambiental la suelen diseñar los actores sociales expertos, mediante la apropiación y el uso de la información científica y técnica (Fischer 2000) o con influencia económica o política. Esto puede constituir una limitante para 
otros actores menos competentes en esos términos, en la toma de decisiones.

Existen instrumentos de política ambiental que incorporan la participación informada, corresponsable e inclusiva, tales como el Convenio de Estocolmo sobre contaminantes orgánicos persistentes (COP). Su objetivo es eliminar o reducir la generación, uso y efectos negativos causados por un grupo de plaguicidas organoclorados, residuos peligrosos, productos industriales y subproductos cuya producción no es intencional (Programa de las Naciones Unidas para el Medio Ambiente, PNUMA 2001). En él se reconoce que los COP son tóxicos, resistentes a la degradación, bioacumulables y trasportados por aire, agua y especies migratorias, depositados lejos del lugar de su liberación, que causan problemas de salud por exposición local, pueden tener efectos en generaciones futuras y que existe la necesidad de tomar medidas de alcance mundial.

En México, desde 1970 se ha producido información que indica la presencia de los COP en medios humanos y ambientales (Albert 1996; Hansen et al. 2006; Castro 2007; Romero et al. 2009), y revelar su uso agrícola y doméstico, su generación en la industria o en la incineración de residuos plantea riesgos que aumentan la vulnerabilidad y la exposición de la población.

Por estas razones, México firmó el Convenio de Estocolmo en 2001, que entró en vigor en 2004, con el que se comprometió a crear el Plan Nacional de Implementación (PNI) para identificar y caracterizar las actividades que originan los COP, y así adoptar medidas efectivas, factibles y aceptables (Secretaría de Medio Ambiente y Recursos Naturales, SEMARNAT 2007). Para ello, el convenio dispone que para la toma de decisiones del PNI se incluya a actores gubernamentales, académicos, industriales, de las organizaciones no gubernamentales (ONG) y de la sociedad civil, a través de mecanismos institucionalizados (PNUMA 2005).

Ese tipo de participación más incluyente fue reconocida desde la Conferencia de Estocolmo (United Nations, UN 1972), y ha tenido repercusiones en acuerdos como el de Río (ibíd., 1992) y en el marco legal mexicano (Diario Oficial de la Federación, DOF 1998); es un elemento ineludible para la democracia (United Nations Economic Commission for Europe, UNECE 1998; Fischer 2000) y favorece 
la legitimidad, la calidad y la capacidad de los procesos decisorios (Dietz y Stern 2008). Sin embargo, cuando se implementa la participación se generan controversias entre quienes tienen información pertinente y entre quienes no, lo cual limita la toma de decisiones en la práctica.

Así pues, las preguntas que motivan esta investigación son: ¿puede el uso y conocimiento de la información determinar si algunos actores tienen mayor influencia en la conformación de instrumentos de políticas ambientales?, o ¿son otras las razones? El propósito de este artículo es examinar el rol simbólico de la información sobre los COP en la participación y en la toma de decisiones para el PNI, como el instrumento rector de la política sobre estos contaminantes.

\section{Enfoque teórico-analítico}

En la elaboración de políticas ambientales, la participación exhibe cuatro fundamentos: a) el legal, cuando sirve como medio para ejercer derechos y obligaciones a un ambiente sano (UNECE 1998); b) el normativo, al legitimar y descentralizar la toma de decisiones, lo que atrae a los afectados del problema ambiental y democratiza el proceso decisorio (O’Faircheallaigh 2010; Fischer 2000); c) el sustantivo, al promover el aprendizaje y mejorar el entendimiento entre participantes (Fiorino 1990) y d) el instrumental, al obtener un suministro informativo y aplicar esas políticas de manera efectiva (Dietz y Stern 2008).

Por otra parte, cuando se implementa la participación hay que considerar algunas de sus características, tales como el nivel de apertura, la forma en que está organizada, la finalidad con que los actores usan la información y la manera de procesarla (Coenen 2009). En este sentido, existe un marco sobre cómo se incluye a los actores, su poder en la toma de decisiones, los objetivos y los mecanismos (encuestas, reuniones, comités), esto indica que el grado de las decisiones varía de una diseminación pasiva de información (manipulación) hasta el involucramiento activo (control ciudadano) (Arnstein 1971); después se redujo a informar, consultar, involucrar, colaborar y a dar- 
les poder de decisión a los participantes (International Association for Public Participation, IAPP 2000).

Sin embargo, en ocasiones la práctica de la participación está condicionada a la posesión, uso y conocimiento de la información científica o pertinente para la toma de decisiones. Esto responde, en parte, al dominio del enfoque tecnocrático (Keller 2009; Fischer 1990; Jasanoff 1990) y, por tanto, a una visión positivista del proceso (Steel et al. 2004).

Lo anterior significa que se emplea más la información que por lo general ostentan los expertos o quienes la producen o poseen, con la finalidad de darle una base técnica a las decisiones. Como esta visión, que ha sido ampliamente cuestionada, de involucrar sólo a los asesores científicos, y la necesidad de las autoridades gubernamentales de consultarlos y mantener vínculos con ellos (Fischer 2000; Jasanoff 1990), se ha buscado incluir un conocimiento derivado de experiencias de actores menos influyentes en aspectos técnicos, económicos y políticos (Innes 1990; Lindblom y Cohen 1979).

La información de los participantes puede validar las decisiones tomadas con anterioridad, en ese entorno técnico, también posicionar intereses o permitir el cumplimiento de compromisos institucionales y marcos legales, y cuestionar así las bases de la participación. Esto sugiere un rol simbólico de la información, cuando diversos actores invitados, participan en una toma de decisiones asociada a la autoridad, las instituciones y los intereses que representan. En esta discusión es en la que se inserta el artículo, al investigar el papel que desempeñan el uso y la apropiación de la información de esos actores. Y, para analizar esta representación simbólica, se subdivide en otros tres roles. En primer lugar, la información constituye un recurso político y estratégico de poder entre los participantes al legitimar sus puntos de vista, decisiones, derechos e intereses cuando la usan, ostentan, rechazan, ocultan o alteran (Mutshewa 2010; Dimitrov 2003), por lo que juega un papel al enmarcar el debate, sustentar e imponer intereses personales o institucionales y al manejarla en torno a la configuración de un plan (Forester 1989; Nelkin 1979).

Para observar y analizar ese rol en la práctica, se deben tomar en cuenta: a) la concentración del poder en las agencias responsables, 
(el) los facilitador(es), y la información que da a conocer o no; b) la concentración del poder entre los participantes más informados (actores que debaten más, opiniones reflejadas en los documentos resultantes, tipos de información de cada actor o sector) y c) los temas, discusiones y argumentos de carácter técnico o político (Mutshewa 2010; Dimitrov 2003; Forester 1989).

En segundo lugar, y como consecuencia de ese ejercicio de poder, se exhibe la exclusión de los menos informados en aspectos técnicos, al minar sus argumentos o al mantener la autoridad de los facultados para debatir y tomar decisiones, lo que polariza el debate, delimita las negociaciones (Hills 2005; Eden 1996; Healy y Ascher 1995) y debilita los fundamentos de la participación. Los aspectos por indagar son: a) las características de la información (confidencialidad, tecnicidad, validez, disponibilidad y acceso); b) las características de los participantes (dominio del tema) y c) los requisitos previos de los invitados, así como recursos (personal, tiempo y presupuesto) y las reglas operativas del ejercicio participativo.

Y, en tercero, la información permite dar cumplimiento al marco legal e institucional y designar responsabilidades a funcionarios y grupos de interés, para demostrar que la toma de decisiones se realiza de forma racional y técnicamente competente, y mostrar así un rol de procedimiento (Hoornbeek 2000). Para examinar cómo se reproduce éste en la práctica, deben considerarse: a) el proceso de participación (objetivos y contexto de la toma de decisiones, la agencia organizadora, los flujos de información y el mecanismo o espacio dispuesto) y b) la selección de participantes (sectores, número por sector, motivaciones y grado de involucramiento).

Si bien se reconoce que el rol simbólico no es la única función que juega la información en la hechura de políticas ambientales, en este artículo interesa explorar su efecto en especial cuando se convierte en un recurso de poder, de exclusión no intencional y de autoridad en la elaboración del PNI, como el instrumento rector de la política sobre los COP en México. Con este enfoque teórico-analítico se busca entender cómo es que los actores y sectores gubernamentales, la iniciativa privada, los académicos y las ONG son involucrados y logran influir en el proceso, aunque eso no siempre les garantiza tomar parte en las decisiones finales. 


\section{Metodología}

Primero se analizó la organización del proceso de participación, para elaborar el PNI, y con ello se contextualizó la metodología, entre noviembre de 2006 y octubre de 2007. La participación para este fin fue diseñada acorde a las guías del PNUMA (2005), mediante una unidad coordinadora de proyecto (UCP), un comité nacional coordinador de seguimiento (CNC), ocho grupos temáticos (GT) y un taller ciudadano (véase figura 1). La SEMARNAT organizó y seleccionó a los

\section{Figura 1}

Estructura de administración del PNI y entrevistados

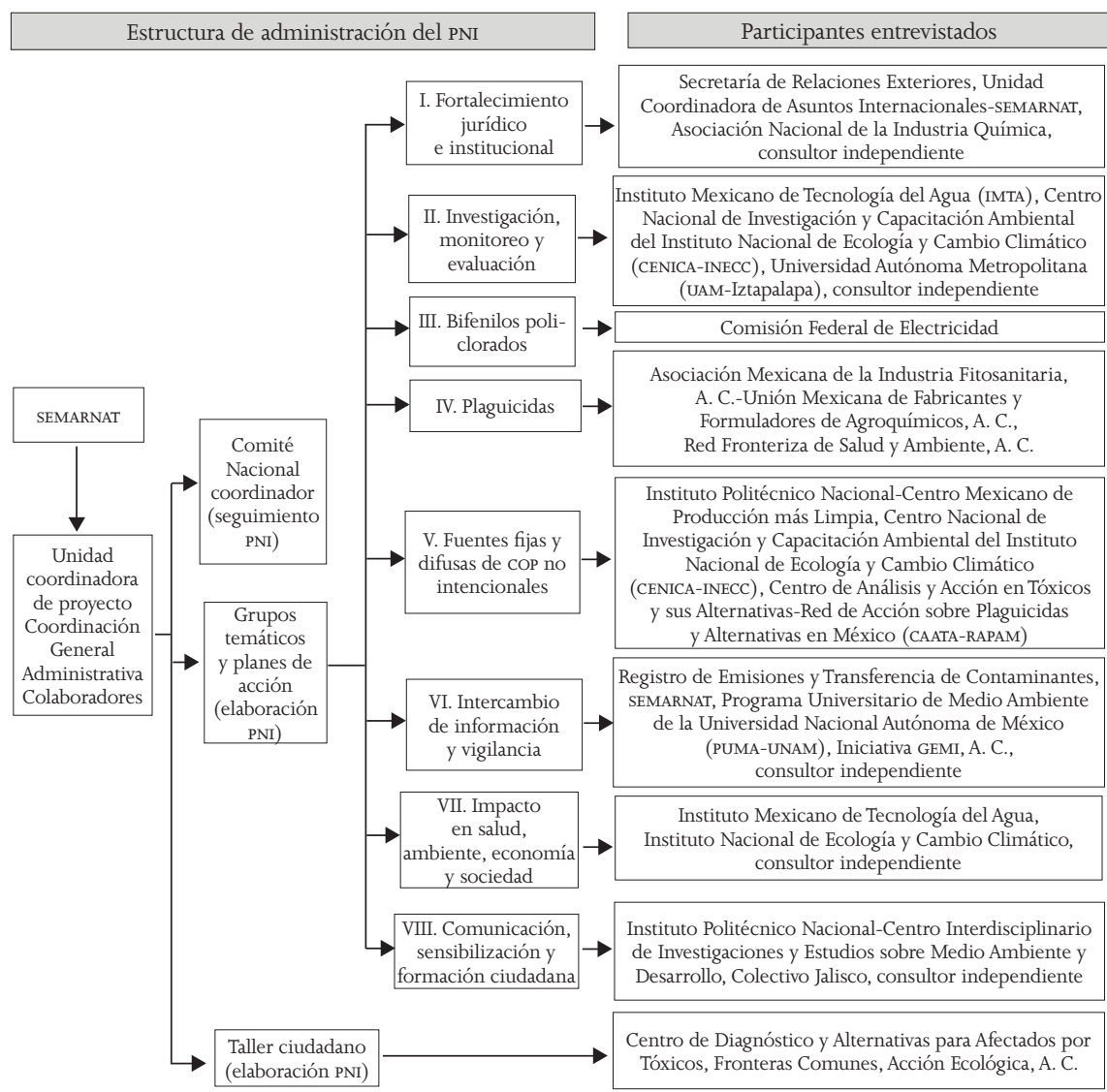

Fuente: elaboración propia, con datos de SEMARNAT (2007). 
participantes, y decidió qué instituciones e intereses debían estar involucrados. A las reuniones se invitaba a cerca de cincuenta actores, de los cuales asistía 70 por ciento.

Con base en lo anterior, la metodología incluyó tres técnicas: a) la revisión documental del escenario histórico de los COP, el Convenio de Estocolmo, la elaboración del PNI y su seguimiento, hasta 2009; b) la observación participante en dos sesiones del CNC (6 de febrero y 22 de mayo de 2009), de este modo se identificaron los participantes, datos personales, comportamientos, categorías de análisis de sus opiniones y el rol simbólico de la información y c) la entrevista semiestructurada.

Primero se seleccionó a los actores de acuerdo con su asistencia, inclusión en los grupos, por sector y el uso que hacen de la información, para lo que se consideró a los siguientes: a) los consultores de los diagnósticos y subplanes; b) los funcionarios públicos que producen información; c) los funcionarios que toman decisiones con esa información; d) los académicos; e) las ONG enfocadas en los COP y f) los representantes de la iniciativa privada que producen o utilizan los COP.

Fueron 36 las entrevistas efectuadas (a dos coordinadores, once funcionarios, ocho ONG, cinco consultores, seis académicos y cuatro representantes de la iniciativa privada) (véase figura 1), con una duración promedio de 45 minutos cada una. A los entrevistados se les describió el proyecto, su propósito y la confidencialidad de los datos. Para las categorías de preguntas se empleó el enfoque teórico-analítico, así como la distinción entre la elaboración del plan y su seguimiento, y se investigaron las opiniones de los involucrados en el tema (no para un público general).

El primer grupo de preguntas explora la situación (actual e histórica) de los Cop a escala nacional. El segundo indaga sobre el rol de la información en la elaboración y alcance de los diagnósticos y subplanes que integran el PNI, la interacción e información de los participantes con consultores, coordinadores y autoridades de la SEMARNAT en los GT y el taller ciudadano.

Después de realizar las entrevistas, los datos se agruparon de acuerdo con: a) las facultades sobre la información de quienes decidieron cómo integrarla al PNI; b) la validación de las decisiones por grupos que sólo asistían o participaban, aunque no siempre incidieran y c) 
actores que sin ser excluidos de manera deliberada, no participan activamente en las discusiones.

El análisis de los datos se apoyó en la teoría fundamentada (Strauss y Corbin 1991) y recomendaciones de Taylor y Bogdan (1987) en tres fases: a) el descubrimiento, que consiste en identificar temas mediante la lectura completa y repetida de los datos antes de iniciar el análisis, desarrollar categorías siguiendo ideas, buscar temas emergentes y leer estudios previos para la interpretación a partir de los supuestos teóricos; b) la codificación, donde se clarifica la comprensión del tema de estudio y c) los resultados, que analizan e interpretan los datos de acuerdo con la pregunta de investigación.

\section{Observaciones y resultados}

La información como recurso de poder en la participación Concentración del proceso en la unidad coordinadora de proyecto

La coordinación técnica de la UCP destacó por su experiencia en temas de sustancias químicas peligrosas. Se elaboraron 15 prediagnósticos para los consultores de los ocho GT con dos intenciones: informar y establecer las bases de diagnósticos más amplios. Diez de los entrevistados reconocieron en la coordinación técnica una autoridad y guía, y tres expresaron que su postura era parcial hacia la participación de las ONG y alineada al ámbito gubernamental, lo que dificultaba la incidencia de este tipo de actores $y$, aunque reconocen la apertura a sus aportaciones, éstas debían tener bases científicas. Así, la coordinación técnica ejerció un poder relevante sobre la delineación del PNI, por un lado como parte de sus funciones asignadas y, por otro, como facilitadora y portadora de información y experiencia.

Ese era mi papel, no tanto que interviniera directamente o diera mi opinión, sino que ponía 'el convenio dice esto, la ley dice esto' y luego hacía este tipo de análisis en los documentos poniendo la información en contexto. En las condiciones en que se hizo fue una labor bastante digna. Considero sinceramente que si yo no lo hubiera hecho, esto no hubiera salido [...] (UCP_12). 
Este control del proceso y de la información representa que quien es considerado una autoridad ejerza poder sobre las decisiones. Aunque hubo consenso, se promovió un ambiente donde las aportaciones debían estar sustentadas jurídica o científicamente. Esto no significa que existiera un ocultamiento de información por parte de la UCP, pero se usó de manera estratégica para cumplir con ciertos objetivos del Convenio de Estocolmo.

Concentración del poder entre los participantes más informados

Por sus fuentes informativas, algunos sectores están mejor y más capacitados para participar, y constituir fuentes de poder (Forester 1989; Mutshewa 2010). En este sentido, el INECC jugó un rol importante al concentrar la información científica sobre los CoP en México. De ahí que la SEMARNAT y otras secretarías del gobierno federal se apoyen en este instituto para tomar decisiones que repercuten en el cumplimiento del Convenio de Estocolmo.

Aunque el sector académico genera su propia información, tiene dificultades para obtener la proveniente del gubernamental, del privado o de la que no se publica, lo que determina que no sean de los grupos que más inciden en las decisiones. Por su parte, el sector industrial genera información técnica con el objetivo de determinar si los COP que producen o manejan representan riesgos para la salud, lo cual les permite definir los inventarios y los procesos que producen los COP. Esto le otorga un lugar preeminente en el debate. En contraste, las ONG generaron su propia información y la han difundido en boletines de prensa, cartas ciudadanas, manuales y guías, y la tradujeron a un lenguaje más sencillo. Gracias a ello hubo portadores que lograron colocarse en las discusiones más técnicas, como en el caso de la RAPAM.

Así se confrontan dos ideas: a) que los científicos no sólo inciden en la conformación de políticas por la supuesta objetividad e imparcialidad que se les adjudica (Jasanoff 1990; Steel et al. 2004; Keller 2009), sino que la participación activa recae en los sectores que manejan mejor la información científica o que poseen la requerida $y$, por tanto, pueden ostentar mayor poder y b) todos estos actores entran a un juego caracterizado por un debate más político que técnico, según los resultados. 
El PNI fue resultado de un debate más político que técnico

Las discusiones con información científica fueron más evidentes en los GT. En el CNC se tomaban decisiones de tipo administrativo, mientras que en el taller ciudadano la participación se limitaba a la consulta sobre lo ya definido en los GT; en el II, V y VII fue más intenso el debate, y en el resto se atendían más las cuestiones jurídicas, estadísticas e ideas para delinear el PNI.

En el GT II, las discusiones radicaron no sólo en garantizar el monitoreo, sino en generar más información para entender los mecanismos de trasporte, acumulación y biodegradación de los COP. Los funcionarios-científicos del INECC sobresalieron de entre los académicos, ya que debatieron en torno a la generación de información confiable y sobre la necesidad de contar con capacidad analítica propia en el país.

[...] asumimos la responsabilidad de tratar de dar respuestas a muchas preguntas que se contestan solamente haciendo monitoreo. Es importante tener datos, es importante generar información que nos ayude a tomar decisiones correctas (funcionario-científico_23).

Las discusiones en el GT vII versaron en torno a hacer diagnósticos sobre los sitios donde se usan o usaron los COP, así como evaluar los niveles de contaminación existentes en el país. Los funcionarioscientíficos sí vieron reflejado este interés en el PNI, quienes han generado datos a la par con los compromisos con la Comisión para la Cooperación Ambiental de América del Norte (CCAAN):

Las recomendaciones que hicimos y la propuesta sobre un programa de investigación y monitoreo de COP, fue tal vez el mejor logro porque es un grupo de sustancias que tienen características muy particulares: análisis diferente, diferentes fuentes de emisión, diferentes efectos toxicológicos (funcionario-científico_31).

En el GT v hubo un debate entre los más informados técnicamente, donde la mayor controversia y diversidad de intereses fue sobre la 
forma de establecer el inventario de emisiones de dioxinas y furanos, así como para reducir sus impactos mediante las mejores tecnologías disponibles y prácticas ambientales. Mientras la academia discutía acerca de actividades, materias primas y temperaturas que generan los COP no intencionales (COPNI), la RAPAM logró colocar información sobre procesos que no contemplaba el instrumental del PNUMA o que se sospechaba estaban mal calculados; puso énfasis en que la mayor generación se debía a las fuentes difusas (quema, incendios) que a las fijas (industria).

El sector industrial intervino al esclarecer la cantidad de emisiones y la tecnología empleada, y aseguró que sí generan COPNI, pero no en las cantidades expuestas por la ONG. No obstante, no se contó con toda la información que podía proporcionar.

En el GT v se evidenció que las decisiones finales recayeron en el consultor, la UCP y la SEMARNAT, de tal modo que pese a que la definición de las fuentes de emisión relevantes es una cuestión técnica, se decidió políticamente:

Ganamos la batalla teórica, pero eso no significó un cambio mayor porque no es suficiente dar la información científicamente más adecuada, darle seguimiento, fundamentarla y no hacer una presión social, política y mediática mayor (ONG_22).

Aunque no sabíamos bien, la decisión fue más política y fue acertada, la incertidumbre no era tan grande. Lo que estamos encontrando es que, en efecto, lo que decía el toolkit era más preciso que usar otros factores de emisión, por este conflicto de intereses (funcionario-científico_14).

Por tanto, la elaboración del PNI fue dominada por elites que producen o poseen la información y que la manipulan de acuerdo a intereses, con decisiones que unas veces privilegian a los funcionarios y otras a los demás grupos de interés pero las finales, a pesar del contenido técnico, quedan en manos de la autoridad. De este modo, una cuestión altamente técnica se torna altamente política, como sugiere Nelkin (1979). 
La información como medio de exclusión de la participación Las características de la información sobre los COP inciden en su conocimiento y uso

La idea de partida fue que el carácter técnico del debate es la limitante más importante de la participación. No obstante, factores como la complejidad de la toma de decisiones, los recursos disponibles, el desconocimiento y la incertidumbre sobre la información también intervienen en que lo que otorgue poder a unos actores y excluya a otros sea ostentar información.

La elaboración del PNI implicó el desconocimiento del comportamiento y generación de los COP y, por ende, de sus riesgos en el país, de su complejidad ante diversos intereses y de incertidumbre cuando la información es confidencial, no confiable o insuficiente, lo que reproduce formas de exclusión no intencional.

Para algunos participantes, temas como los COPNI son una cuestión incomprensible y que requiere formación previa, ya que deben aprobar decisiones respecto a sus efectos:

Cada vez que tenemos alguna reunión aprendemos más porque decimos: ¿cómo que esa sustancia hace esto y es dañina? [...] Sobre todo los [temas] técnicos, de COP intencionales y no intencionales, dices: ¿qué es eso, qué con las dioxinas y los furanos? Son temas bastante complicados que en una sola reunión no entiendes. Nuestra formación es más política (funcionario público_20).

Me costaba trabajo entender el ciclo de manejo de los cop: cuáles son las fuentes, quién y cómo las está manejando, cómo es que se emiten, por qué va ligado a la industria. A lo mejor un producto que consumimos ya los trae y no sabemos. No entendía cómo los regulaba el país (consultor_5).

Cinco de los entrevistados reconocieron que persiste una masa crítica reducida, y que falta información para tomar decisiones con la menor incertidumbre. No obstante, la industria es el principal poseedor de información para los diagnósticos y, aunque sí aportó datos, se mostró renuente a hacerlo al considerar que tendría efec- 
tos en el acatamiento de nuevas regulaciones o al dejar que el gobierno manejara información estratégica que tendría implicaciones económicas.

Ostentar información técnica no limita el uso de otros tipos de conocimiento

Recrear un diálogo técnico ha sido parte de la participación en contextos tecnocráticos (Forester 1989; Fischer 2000). En este caso, sólo en algunos GT se discutió información técnica, y en otros cuestiones sobre vacíos y traslapes del marco jurídico, inventarios de los COP o estrategias de comunicación de riesgos. Si bien la UCP proporcionó algunas bases, esto no garantizó un debate entre expertos y con toda la información requerida para tomar decisiones. Aunque los entrevistados reconocieron la importancia de incluir la experiencia de la sociedad civil, al menos con las ONG, y así validar las decisiones, se trata de foros donde difícilmente participa quien no tiene las capacidades o facultades exigidas:

Los involucrados son desde los que hacen la política, los que hacen investigación, como los que usan los productos y los que están en contacto con esos productos. Entonces, definitivamente tienen que estar incluidos todos los actores, la sociedad tiene que estar enterada, si no no tiene sentido. ¿Para qué hacemos una investigación con muchos resultados, sabemos cuáles son los efectos, si esto no se traduce en acciones y no se previene a la sociedad de cómo debe de enfrentarlo? (académico_19).

$[\ldots]$ quieren que nos nombremos como expertos en el sentido de que tengamos una calificación para estar ahí. Las ONG no estamos ahí porque seamos expertos, sino porque compartimos una visión. Para nosotros es importante que se tomen en cuenta otras necesidades: mujeres, trabajadores, niños, procesos de difusión y participación pública. Esa información que se genera, poder usarla y hablar con un ama de casa y explicarle con diferentes herramientas qué le está afectando (ONG_1).

De este modo, aunque exista interés de algunos para incluir el conocimiento local, éste se restringe cuando no representa informa- 
ción útil en la toma de decisiones. Esto implica que aunque se crean espacios para participar, ostentar información relevante es un requisito para la inclusión y, por tanto, constituye un recurso de poder, mas no es una limitante. Por tanto, está ausente un mecanismo eficaz que homogenice la información y su comprensión para los participantes.

La información es ostentada como instrumento para validar la participación

El carácter técnico de la información no es limitante de la participación

La autoridad que ejerció la UCP la habilitó para decidir el tipo de actor que sería involucrado, además de los elegidos por la SEMARNAT. Se invitó a especialistas que trabajan en la gestión de las sustancias químicas, aunque varias de las secretarías del gobierno federal no participaron activamente (como la Secretaría de Agricultura, Ganadería, Desarrollo Rural, Pesca y Alimentación o la Secretaría de Salud).

A los funcionarios especialistas se les reconoce su experiencia sobre inventarios de emisiones y la investigación del trasporte de los COP; entre ellos se encuentran los del IMTA, del CENICA y del INECC cuyas atribuciones les confieren autoridad en el tema e inclusive se visualizan como invitados "obligatorios" a participar en el PNI.

El resto de actores también fueron invitados, pero no por convocatoria, representatividad geográfica o poseer experiencia directa de la problemática:

Como se trataba de materias técnicas, a lo que se recurrió fue al famoso directorio de 'quién es quién' y entonces se trató de invitar a personas que sabían de esas materias. Se les invitó y los que aceptaron son a los que se incluyó. Fueron invitados por cartas $[\ldots]$ (UCP_0).

Ejemplo de ello fue la comunidad académica, como aquéllos que ya habían trabajado con la SEMARNAT, por investigación en ciencia y tecnología, y para incluir visiones sociales, como El Colegio de México. En cambio, para el sector empresarial, hubo una invitación directa a instituciones, cámaras y a sus representantes que ya han trabajado en otros foros ambientales, a fin de suministrar información 
sobre plaguicidas COP, bifenilos policlorados (BPC) o COPNI, y otorgar su aprobación al plan.

Las ONG se mostraron críticas por la forma en que fueron incorporadas (a título personal) y por el hecho de que, al igual que el sector académico, fueron minoría. Sin embargo, aceptan que deben aprovechar el espacio. Estas ONG consideran que su invitación se debe a que la RAPAM asiste a reuniones sobre el convenio, y porque éste establece que la sociedad civil debe participar para validar las decisiones gubernamentales.

Esto evidencia que, para que la participación sea exitosa, la cualidad técnica que proporciona un reconocimiento o manejo del tema es primordial para mantener una deliberación consensuada y razonada. Por tanto, es sólo un requisito para ser invitados, mas no una limitante cuando varios de ellos no son expertos o sólo poseen la información pertinente. La participación ocurre aun sin esa condición, y no en todos los casos fue un factor para incidir en las decisiones.

El carácter técnico de un participante en la consulta no es un indicativo de cómo se desarrolla un proceso más político que técnico. Por ello no debe ser una limitante para otros grupos que no pueden verse representados; en realidad, juega más un rol como parte de la percepción que se tiene de los expertos. Su incorporación obedece más a propósitos de validación de las decisiones, y de demostrar que en la elaboración del PNI se invitó a actores clave y especialistas en el tema.

Límites al esquema racional de participación

La participación e inclusión a los GT fue abierta para los actores ya seleccionados, a fin de aportar información y evaluar los diagnósticos y los subplanes de acción de los consultores. Sin embargo, en el número de invitados no fue equitativo (fueron más funcionarios y empresas que ONG y académicos), además de que faltaron recursos para traer a los actores foráneos a las reuniones en la Ciudad de México. Estos también son obstáculos claros para la participación, ya que se promueve el centralismo y se restringe a los menos representados y, por lo tanto, su incidencia en las decisiones.

Por un lado, los flujos de información entre consultores y la UCP con los participantes del comité y los GT propiciaban diálogos con los más propositivos, y se restringía para los que sólo podían enviar su 
información vía electrónica. Aun así, revisar los documentos fue clave para saber si se estaban incorporando las opiniones.

En otros casos, la UCP y la SEMARNAT privilegiaban a los actores con los que tenían más relación o de los que buscaban mayor participación, como el sector industrial o los mismos funcionarios, generadores o no de información.

Si bien se plantea que el rol de la información técnica tiene mayor peso en la conformación del PNI, en este caso la apatía política, los intereses que entran en competencia, y las restricciones institucionales y organizacionales (tiempo y recursos) son limitantes importantes del esquema racional de toma de decisiones. Un efecto claro es que, al no designarse presupuesto suficiente y centralizar las decisiones, se restringe la capacidad gubernamental para promover una participación incluyente, corresponsable e informada.

El alcance real de la participación

La participación en el PNI cumplió los objetivos de informar, consultar e involucrar a los grupos de interés en las decisiones gubernamentales mediante el CNC y los GT, y sólo en una consulta pública al resto de la sociedad, representada con las ONG y otros grupos de interés, a través del taller ciudadano. En estas condiciones, la participación es de las menos efectivas (Arnstein 1971; IAPP 2000).

El sector académico se identificó a sí mismo como portador de información y opiniones válidas, aunque sujetas al diseño ya dispuesto del PNI y a las decisiones de gobierno, es decir, a la incorporación y la consulta. Se mostró crítico de la forma en que su participación se ha reducido a reuniones informativas sobre las actividades de la SEMARNAT para dar cumplimiento al convenio. El sector empresarial se reconoció como aliado histórico en la creación de la legislación y otros planes, y como poseedor de información para los inventarios, pero también atenido a restricciones económicas y operativas para ejecutar las acciones del PNI. Esta elite ostenta y manipula estratégicamente la información al participar y decidir junto con la autoridad:

A lo mejor nosotros mismos tomábamos la 'batuta' en eso junto con las dependencias, y dependencias a nivel federal, que eran las que participaban en ese entonces (industria_15). 
En contraste, para las ONG, el comité y los GT funcionaron como formas de establecer el diálogo y la participación, pero no como mecanismos de concertación para incidir en las decisiones. Aunque en el taller ciudadano tuvieron mayor presencia, la visión de las ONG es que se limitó a exhibir que se incluye a la sociedad en planes gubernamentales y que existe un trabajo armonioso con los otros sectores:

Nos queda claro que tenemos que estar en donde se hacen las políticas públicas. No le podemos llamar una participación efectiva en la toma de decisiones, porque eso está muy lejano. Deben respetar los espacios, los tiempos, las visiones de todos los que participan en la discusión. De nada sirve que estén todos los sectores y las instituciones cuando no va a ser válida la participación de las ONG, sólo la de los demás. Fue una forma de llenar el requisito de que sí hubo participación (ONG_1).

Para los funcionarios públicos fue un proceso trasparente e incluyente, pero también sujeto a intereses. Según su postura, la idea es crear un espacio participativo racional y donde se discuta la información verídica y científica. En la elaboración del PNI, instancias de la SEMARNAT y del INECC tuvieron mayor contribución y peso en las decisiones.

Entre funcionarios que no realizan investigación, la generación de políticas recae en el gobierno, el que debe liderarlas, y apoyarse en la participación equilibrada con los grupos de interés, es decir, limitada a obtener retroalimentación de manera directa a través de la consulta, y que pueda orientar las decisiones que tomen las secretarías involucradas.

La participación en el seguimiento al PNI se limitó a que la SEMARNAT sólo informó las decisiones ya tomadas, lo que redujo el objetivo de lograr corresponsabilidad entre los sectores e incluso entre sus mismos órganos desconcentrados, y restringió la participación trasversal con otras secretarías, de por sí difícil de lograr en la elaboración del PNI:

El mayor peso fue a la hora de votar. Al final, mucha gente percibió que esta es una cancha del gobierno, y que es la pelota del go- 
bierno porque al Estado le toca resolver el problema, y los demás eran invitados nada más para que se les informe [...] (UCP_4).

La participación queda así limitada al involucramiento, a la consulta y a informar el avance de acciones ya contempladas, lo que la vuelve simbólica (Arnstein 1971; IAPP 2000), como también al rol que juega la información cuando el proceso está condicionado a que quienes la aporten sean especialistas, o quienes participen la empleen estratégicamente, sean o no expertos, impongan intereses, den poder a unos y excluyan a otros. También es un factor que otorga autoridad a quien la ostenta, por la cual es invitado a un proceso en el que no todos inciden.

\section{Conclusiones}

El caso de la elaboración y seguimiento del PNI sobre los COP fomenta una comprensión de la forma en que ocurre la participación social en la práctica, frente a lo que es en la teoría. En este sentido, el enfoque teórico-analítico es útil para entender cómo el proceso de toma de decisiones de políticas ambientales se presta a juegos de poder sobre la información que ostentan los actores más informados o competentes en los ámbitos técnico y legal, en lugar de sólo prestar atención a los que política y económicamente son más poderosos.

No obstante, entre los participantes más relevantes se encuentran: a) los funcionarios-científicos, con facultades en la gestión de sustancias químicas y residuos peligrosos, o que velan por el cumplimiento de los acuerdos internacionales; b) el sector industrial, que comercializó o usó los COP (plaguicidas, BPC) o que genera los COPNI y c) las ONG, que consiguen debatir con información validada científicamente promoviendo así un escenario de toma de decisiones más político que técnico.

Ostentar la información sobre los COP resulta una condición para el involucramiento de actores y de validación del PNI, a partir de la apreciación que se tiene de varios de ellos como autoridades en la materia. Promover debates entre unos expertos en el tema y otros que no lo son tiene un fuerte efecto en validar decisiones en un 
esquema tecnocrático y supuestamente democrático, que incluye de forma parcial a los sectores de acuerdo con reglas formales, pero no en hacerlos parte de un círculo de tomadores de las decisiones finales.

En realidad, la condición sobre el carácter técnico requerido para el diálogo determinó, primero, quiénes serían los invitados a participar y, segundo, les confirió autoridad para opinar y ostentar información relevante. Esto evidencia que en la elaboración de planes de gobierno, un tema técnico buscará ser mejor discutido por expertos, lo que cierra el debate a los menos informados, aun cuando el manejar la información pertinente es uno de los factores que moldean la participación y los resultados de la elaboración de políticas públicas ambientales.

Las decisiones sobre el PNI no fueron puramente técnicas ni encabezadas por expertos externos a la esfera gubernamental, sino políticas, sociales y económicas, sujetas a valores que entran en conflicto, con repercusión nacional e internacional. Esto sugiere un cuestionamiento a la orientación positivista y da paso a otras necesidades de información.

La exclusión es la consecuencia de exhibir saberes e intereses institucionales, fundamentados científica o económicamente, en donde no todos tienen las mismas oportunidades para participar y más aún de situarse en la toma de decisiones finales y, tal como lo exponen Arnstein (1971) y Forester (1989), produce acuerdos pasivos que validan simbólicamente las decisiones.

De este modo, "apropiarse" la información no es el único factor que delimita la participación o que incide en las decisiones y, por tanto, no garantiza que los intereses de todos los participantes, expertos o no, predominen en la conformación de una política tecnificada. Los hallazgos apuntan a la existencia de otras limitantes de la participación, cuyos efectos son más importantes que el carácter técnico como requisito, además de los intereses que entran en competencia o la falta de presupuesto para apoyar a los participantes foráneos. Como resultado, el PNI es un condensado de propuestas generadas por los consultores, organizadores y la autoridad en ese momento, bajo esas limitaciones, en un contexto de desconocimiento del tema, de falta de masa crítica y, a la vez, de acuerdo con la información disponible. 
En la elaboración del PNI, la información jugó un rol simbólico como recurso de poder para imponer intereses entre participantes en las negociaciones, y como elemento para validar las decisiones finales, e incidir así en la dinámica entre los menos informados y entre los que, aunque informados (de cualquier grupo de interés), no logran incidir en las decisiones manteniendo un desequilibrio entre perspectivas sobre la forma de implementar el Convenio de Estocolmo en el país.

Así, lo que está en juego, en un plan que sólo es orientativo y no programático en el caso de los funcionarios, es que se dé seguimiento a los acuerdos internacionales, que se establezcan regulaciones de mayor peso jurídico (como una ley general de sustancias químicas) y captar recursos del exterior para aplicar esos acuerdos. Para los funcionarios-científicos es fortalecer programas de monitoreo, para producir información confiable y definir si existen riesgos. Este sector se constituye como la red principal de relaciones de poder e información con la cual se diseña y ejecuta la política sobre los cop.

Aunque la comunidad académica continúa midiendo niveles de los COP en varios medios, no siempre son personajes activos de la difusión de sus riesgos. Esto ha quedado en manos de las pocas ONG en el país dedicadas a estos temas, cuyo mayor interés ha sido difundir los impactos negativos al ambiente, a la salud humana y a las generaciones futuras, mediante políticas dirigidas a eliminar, más que a reducir los riesgos del uso y generación de sustancias químicas dañinas.

La iniciativa privada está interesada en la implementación del Convenio de Estocolmo, porque constituye una fuente de vigilancia y acatamiento jurídico futuro. Para este sector, los riesgos a la salud humana y ambiental son minimizados si su manejo, trasporte y almacenamiento son adecuados.

Este artículo es una expresión de lo que otros autores han estudiado sobre las formas de actuación entre la sociedad y el gobierno o el por qué la participación informada, incluyente y corresponsable no siempre se materializa en la práctica. El aporte reside en ver que la apropiación del conocimiento y uso de la información en la toma de decisiones tiene un rol que poco se revela, si no se profundiza en el análisis del contexto y de las respuestas sobre cuestiones ambientales como la de los cop en el ámbito nacional. 
Recibido en noviembre de 2013 Aceptado en agosto de 2014

\section{Bibliografía}

Albert, Lilia. 1996. Persistent pesticides in Mexico. Reviews of Environmental Contamination and Toxicology (147): 1-44.

Arnstein, Sherry R. 1971. A ladder of citizen participation. Journal of Royal Town Planning Institute: 2-6.

Castro, José. 2007. Recopilación de información sobre sustancias tóxicas prioritarias en México. Canadá: CCAAN.

Coenen, Frans (editor). 2009. Public participation and better environmental decisions. The promise and limits of participatory processes for the quality of environmentally related decision-making. Springer.

Dietz, Thomas y Paul Stern (editores). 2008. Public participation in environmental assessment and decision making. Panel on public participation in environmental assessment and decision making. Washington, D.C.: National Academies Press.

Dimitrov, Radoslav S. 2003. Knowledge, power, and interests in environmental regime formation. International Studies Quarterly 47 (1): 123-150.

DOF. 1988. Ley General del Equilibrio Ecológico e Impacto Ambiental. 28 de enero de 1988.

Eden, S. 1996. Public participation in environmental policy: considering scientific, counter-scientific and non-scientific contributions. Public Understanding of Science 5: 183-204.

Fiorino, Daniel J. 1990. Citizen participation and environmental risk: a survey of institutional mechanisms. Science,Technology and HumanValues (15): 226-243. 
Fischer, Frank. 2000. Citizens, experts, and the environment: the politics of local knowledge. Londres: Duke University Press. 1990. Technocracy and the politics of expertise. Newbury Park: Sage Publications.

Forester, John. 1989. Planning in the face of power. Berkeley: University of California Press.

Hansen, Anne, M., Manfred van Afferden, Mariana Villada Canela, Luis F. Sánchez. 2006. Scoping study for the evaluation of the national program of monitoring and environmental assessment in Mexico (proyecto de investigación). IMTA, CCAAN, Canadá.

Healy, Robert G., y William Ascher. 1995. Knowledge in the policy process: incorporating new environmental information in natural resources policy making. Policy Sciences 28: 1-19.

Hills, Sarah. 2005. Falling through the cracks: limits to an instrumental rational role for environmental information in planning. Environment and Planning A (37): 1263-1276.

Hoornbeek, John. 2000. Information and environmental policy: a tale of two agencies. Journal of Comparative Policy Analysis: Research and Practice 2: 145-187.

IAPP. 2000. Espectro de participación pública. http://www.iap2. org/index.cfm (2013).

Innes, Judith E. 1990. Knowledge and public policy: the search for meaningful indicators. New Brunswick: Editorial Transaction.

Jasanoff, Sheila. 1990. The fifth branch: science advisers as policymakers. Cambridge: Harvard University Press.

Keller, Ann Campbell. 2009. Science in environmental policy: the politics of objective advice. Cambridge: The MIT Press. 
Lindblom, Charles E., y David K. Cohen. 1979. Usable knowledge: social science and social problem solving. New Heaven: Yale University Press.

Mutshewa, Athulang. 2010. The use of information by environmental planners: a qualitative study using grounded theory methodology. Information Processing and Management (46): 212-232.

Nelkin, Dorothy. 1979. Controversy: politics of technical decisions. Beverly Hills: Sage Publications.

O'Faircheallaigh, Ciaran. 2010. Public participation and environmental impact assessment: purposes, implications and lessons for public policy making. Environmental Impact Assessment Review 30 (1): $19-27$.

PNUMA. 2005. Cuestiones que se someterán al examen de la Conferencia de las Partes o respecto de los cuales deberá adoptar una decisión: planes de aplicación. Anexo: guía para el desarrollo de un plan nacional de aplicación del Convenio de Estocolmo. Documento UNEP/POPS/COP.2/INF/ 7 .

. 2001. Convenio de Estocolmo sobre Contaminantes Orgánicos Persistentes, documento publicado por la Secretaría del Convenio de Estocolmo sobre Contaminantes Orgánicos Persistentes. 22 de mayo.

Romero, Teresita, Cristina Cortinas y Víctor Gutiérrez. 2009. Diagnóstico nacional de los contaminantes orgánicos persistentes en México. México: SEMARNAT, Instituto Nacional de Ecología.

SEMARNAT. 2007. Plan Nacional de Implementación del Convenio de Estocolmo. Primera edición en versión electrónica. http://siscop. ine.gob.mx/novedades/pni_mexico.pdf (2013).

Steel, Brent, Peter List, Denise Lach y Bruce Shindler. 2004. The role of scientists in the environmental policy process: a case study from the American west. Environmental Science \& Policy 7: 1-13. 
Strauss, Anselm L., y Juliet Corbin. 1991. Basics of qualitative research: grounded theory procedures and techniques. Newbury Park: Sage Publications.

Taylor, Steve J., y Robert Bogdan. 1987. Introducción a los métodos cualitativos de investigación: la búsqueda de significados. México: Paidós.

UN.1992. Agenda 21 y Declaración de Río.

1972. United Nations Conference on the Human Environment. Nueva York.

UNECE. 1998. Convention on access to information, public participation in decision-making and access to justice in environmental matters. Aarhus, Denmark. 25 de junio. www.unece.org/env/pp/ documents/cep43e.pdf 
